\title{
MULTILEVEL SCPC SYSTEM DESIGN
}

\author{
M. Horstein and D. T. LaFlame \\ Space and Communications Group \\ Hughes Aircraft Company \\ Culver City, California
}

\begin{abstract}
A method of assigning carrier levels in an SCPC system with mixed earth station G/Ts is developed which optimizes system performance for uniformly spaced and randomly assigned carrier frequencies. The optimum transponder backoff is shown to be identical to that for a system of uniform carriers in which the (common) earth station $(\mathrm{G} / \mathrm{T})^{-1}$ is a weighted average of the different $(\mathrm{G} / \mathrm{T})^{-1}$ values in the mixed system. With the transponder backoff determined, the carrier level to be transmitted to each station type is simply expressed in terms of the station $\mathrm{G} / \mathrm{T}$.
\end{abstract}

\section{INTRODUCTION}

It is widely recognized that single channel per carrier (SCPC) transmission can provide considerably greater satellite capacity in a system characterized by light-traffic routes than is available through the traditional FDM/FM method of modulation. This is accomplished, first, through an increase in the number of voice channels per transponder and, second, from the nearly 100 percent utilization factor that results from the demand assignment of satellite circuits.

SCPC transmission affords another advantage, however, which is seldom exploited. Since each transmitted carrier is generally received by only a single earth station, its power level can be adjusted in accordance with the $\mathrm{G} / \mathrm{T}$ of the receiving station Application of this simple idea in a power limited situation can result in a significant increase in transponder capacity over that achievable with a system of uniform carriers. Alternatively, multilevel transmission can be utilized to achieve the bandwidth limit on capacity with smaller earth stations than would be possible in a uniform carrier system.

A mixture of earth station types might be encountered in a number of system contexts. In a thin-route system, for example, one or more stations are typically located in the economic and/or political centers of a country or region. These stations, which serve as terminals for a large number of thin routes, assume the form of major installations. The much more 
numerous outlying stations are of simpler design. Traffic flow is predominantly between large and small stations, with a much smaller amount (if any) between pairs of small stations. Traffic between pairs of large stations is usually handled separately.

A second example is provided by a network of mixed stations dominated by traffic between pairs of the largest stations in the system. This traffic pattern could develop from an initial configuration in which a transponder is devoted to traffic among the larger stations. The latter would normally be located at major population centers. Lesser population centers would subsequently be included through the introduction of smaller earth stations. In this way, a network could evolve requiring several different carrier sizes, one for each distinct earth station design. The key feature of such a system is that, regardless of the number of smaller earth stations, the great majority of carriers are received by the larger stations. As a consequence, most of the carriers can be transmitted at a relatively low level, with only those carriers directed at the smaller stations requiring a higher transmitter power.

The multilevel transmission technique discussed in this paper is based on the availability of a dedicated SCPC transponder. This is an increasingly common situation with the proliferation of national and regional satellite systems in either an operational or planning stage.

Carrier frequencies are assumed to be assigned in a random manner, so that the distribution of carrier levels is the same for all frequencies. This randomness arises quite naturally in the context of a demand assignment system, since it is a simple matter to design the system software to meet each new circuit request with an essentially random choice of frequencies.

It will be shown, as a general property of such a system, that the transponder backoff leading to the optimum set of carrier levels is the same as that for a system of uniform carriers in which the (common) earth station $(\mathrm{G} / \mathrm{T})^{-1}$ is a weighted average of the different $(\mathrm{G} / \mathrm{T})^{-1}$ values in the mixed system. Once the optimum transponder backoff has been found, the carrier level to be transmitted to each station type is readily determined in terms of the actual G/T for that station.

\section{GENERAL MULTILEVEL SYSTEM}

The carrier frequencies in this system are uniformly spaced across the transponder bandwidth. The analysis is performed for a fully loaded transponder - that is, a situation in which all carrier frequencies have been assigned. It is assumed that this set of assignments is the outcome of a process in which each new circuit request results in a selection of two frequencies, each chosen with equal probability and independently of the other, from those 
frequencies not yet assigned. The fact that, in practice, frequencies are usually selected in prearranged pairs does not materially affect the results of this analysis. It is only necessary, when two different carrier levels are involved, that the higher powered carrier be assigned with equal probability to either frequency.

The total intermodulation (IM) power generated by the ensemble of carriers is a function only of the transponder backoff. (This was demonstrated in Reference 1 for the case of two carrier levels; extension to multiple levels is straightforward. ) Because of the large number of carriers accessing the transponder, the percentage of carriers at each power level is assumed to remain constant. Consequently, the transponder backoff is also constant. It follows that, for a specified set of carrier levels, the randomness in assigning frequencies guarantees that the average IM power at a given frequency is equal to the fixed IM power at the same frequency in a system of uniform carriers operating at the same backoff.

The optimum set of carrier levels is defined to be that set which maximizes the minimum carrier-to-noise ratio (CNR) over the transponder bandwidth, provided that the CNR requirements are identical at all station types. If this is not the case, the optimum set of carrier levels maximizes the minimum CNR for any single station type, with the prescribed CNR differences being maintained between maximin values for different station types. The set of relative carrier levels thereby established maximizes the transponder capacity in a power limited situation, in which case a reduced number of carrier frequencies are chosen at random from the total set of transponder frequencies. In a bandwidth limited situation, the system power margin is maximized for the given combination of earth stations.

In deriving the optimum set of carrier levels, it is assumed that the minimum CNR occurs at the center carrier positions). This is a valid assumption provided that 1) the IM spectrum peaks at the center carrier frequency, a condition which is satisfied for a uniformly spaced set of equal amplitude carriers, and therefore by the average IM spectrum resulting from a random set of carrier assignments, and 2) transponder end effects are not significant. The latter condition refers to the possibility of transponder "multipath" and/or IM interference from an adjacent transponder degrading the CNR of the end carriers to a value lower than that at band center. It will be assumed that the bandwidth spanned by the carriers is chosen, in conjunction with the carriers occupying the adjacent transponder, so that this does not happen.

Assume that there are $S$ station types and let $\gamma_{\mathrm{ij}}$ represent the fractional system traffic between stations of types $\mathrm{i}$ and $\mathrm{j}, 1 \leq \mathrm{i} \leq \mathrm{j} \leq \mathrm{S}$. The ordering of station types is arbitrary. The fractional number of carriers received by stations of type $\mathrm{k}$ (i.e. , the fractional number of carriers of type $\mathrm{k}$ ) is given by 


$$
\gamma_{k} \triangleq \frac{1}{2}\left[\sum_{i=1}^{k} \gamma_{i k}+\sum_{i=k}^{S} \gamma_{k i}\right]
$$

Let

$$
\begin{aligned}
\mathrm{P}_{\mathrm{k}} & =\underset{\text { repeater input power, normalized to the saturation power, in each carrier of }}{\text { type }} \\
\mathrm{r}_{\mathrm{k}} & =\mathrm{P}_{\mathrm{k}} / \mathrm{P}_{1} \\
\mathrm{C}_{\mathrm{k}} & =\text { normalized repeater output power in each carrier of type } \mathrm{k} \\
\mathrm{C}_{\mathrm{a}} & =\text { normalized average power per carrier at repeater output } \\
& =\sum_{\mathrm{k}=1}^{S} \gamma_{\mathrm{k}} \mathrm{C}_{\mathrm{k}}
\end{aligned}
$$

It can be shown (Reference 1) that, with a large number of carriers, the relative carrier levels are unaffected by the nonlinear transponder characteristic. Therefore,

$$
\begin{gathered}
\mathrm{C}_{\mathrm{k}}=\mathrm{C}_{1} \mathrm{r}_{\mathrm{k}} \\
\mathrm{C}_{\mathrm{a}}=\mathrm{C}_{1} \sum_{\mathrm{k}=\mathrm{l}}^{\mathrm{S}} \mathrm{\gamma}_{\mathrm{k}} \mathrm{r}_{\mathrm{k}}
\end{gathered}
$$

The uplink thermal noise in the band of a single carrier will be denoted by $\mathrm{N}_{\mathrm{u}}$; the downlink thermal noise at a station of type $\mathrm{k}$, by $\mathrm{N}_{\mathrm{dk}}$. The corresponding receive system noise temperatures are represented by $\mathrm{T}_{\mathrm{u}}$ and $\mathrm{T}_{\mathrm{dk}}$. The uplink and downlink carrier-tothermal noise ratios for a carrier of type $\mathrm{k}$ can be written as"

$$
\frac{P_{k}}{N_{u}}=\frac{P_{k}}{k T_{u} B}=\frac{C_{k}}{k T_{u} B G}
$$

\footnotetext{
${ }^{*} \mathrm{~T}_{\mathrm{dk}}$, and therefore $\mathrm{N}_{\mathrm{dk}}$, is referred to the transponder output (i.e., to the point at which $\mathrm{C}_{\mathrm{k}}$ is defined). It reflects the $\mathrm{G} / \mathrm{T}$ for a station of type $\mathrm{k}$, as well as variations in satellite transmit antenna gain and path loss.
} 


$$
\frac{\mathrm{C}_{\mathrm{k}}}{\mathrm{N}_{\mathrm{dk}}}=\frac{\mathrm{C}_{\mathrm{k}}}{\mathrm{k} \mathrm{T}_{\mathrm{dk}} \mathrm{B}}
$$

where $\mathrm{G}=\mathrm{C}_{\mathrm{k}} / \mathrm{P}_{\mathrm{k}}, \mathrm{k}=1,2, \ldots, \mathrm{S}$, is the transponder gain and $\mathrm{B}$ is the carrier noise bandwidth.

If $\mathrm{IM}_{\mathrm{c}}$ denotes the average IM power falling on the center carrier(s), the CNR for a carrier of type $\mathrm{k}$ at the center of the transponder is given by

$$
\left(\frac{C}{N}\right)_{k} \triangleq \frac{C_{k}}{k B\left(T_{u} G+T_{d k}\right)+I M_{c}}
$$

Use of Equations 2 and 3 in Equation 6 leads to

$$
\left(\frac{C}{N}\right)_{k}=\frac{{ }_{k} X_{k}}{\sum_{i=1}^{S} \gamma_{i}{ }^{r}{ }_{i}}
$$

where

$$
x_{k}=\frac{C_{a}}{k B\left(T_{u} G+T_{d k}\right)+I M_{c}}
$$

Note that $\mathrm{X}_{\mathrm{k}}$ is a function of the transponder backoff through $\mathrm{C}_{\mathrm{a}}, \mathrm{G}$, and $\mathrm{IM}_{\mathrm{c}}$, but is independent of $r_{i}, i=1,2, \ldots, S$.

Provision will be made for different CNR requirements at different station types. If $\beta_{\mathrm{k}}$, $\mathrm{k}=1,2, \ldots, \mathrm{S}$, is the set of factors specifying the relative CNR values such that

$$
\left(\frac{\mathrm{C}}{\mathrm{N}}\right)_{\mathrm{k}}=\frac{1}{\beta_{\mathrm{k}}}\left(\frac{\mathrm{C}}{\mathrm{N}}\right)_{1}, \mathrm{k}=1,2, \ldots, \mathrm{S}
$$

the optimum set of carrier levels is found by maximizing $(\mathrm{C} / \mathrm{N})_{1}$ subject to Equation 9 . Equivalently, it is necessary to maximize

$$
\begin{array}{r}
F\left(P_{\text {in }}, r_{2}, \ldots, r_{S}, \lambda_{2}, \ldots, \lambda_{S}\right) \triangleq\left(\frac{C}{N}\right)_{1} \\
+\sum_{k=2}^{S} \lambda_{k}\left[\left(\frac{C}{N}\right)_{1}-\beta_{k}\left(\frac{C}{N}\right)_{k}\right]
\end{array}
$$


where $\mathrm{P}_{\text {in }}$ is the transponder input backoff and $\lambda_{2}, \ldots, \lambda_{\mathrm{S}}$ form a set of S-1 Lagrange multipliers.

Setting the partial derivative of $\mathrm{F}$ with respect to $\lambda_{\mathrm{k}}$ equal to zero and substituting from Equation 7 yields

$$
r_{k}=\frac{x_{l}}{\beta_{k} x_{k}}, k=2,3, \ldots, s
$$

Substitution of Equation 7 into Equation 10, followed by differentiation with respect to $r_{k}$, results in

$$
\frac{\partial F}{\partial r_{k}}=-\frac{\gamma_{k} F}{\sum_{i=1}^{S} \gamma_{i} r_{i}}-\frac{\lambda_{k} \beta_{k} X_{k}}{\sum_{i=1}^{S} \gamma_{i}{ }^{r}}, k=2,3, \ldots, s
$$

However, with Equation 9 satisfied, $F=(C / N)_{1}$, so that

$$
\frac{\partial F}{\partial r_{k}}=-\frac{\gamma_{k} x_{1}}{\left(\sum_{i=1}^{S} \gamma_{i}{ }^{r}\right)^{2}}-\frac{\lambda_{k} \beta_{k} x_{k}}{\sum_{i=1}^{S} \gamma_{i}{ }^{r}{ }_{i}}, k=2,3, \ldots, s
$$

Setting $\partial \mathrm{F} / \partial \mathrm{r}_{\mathrm{k}}=0$, solving for $\lambda_{\mathrm{k}}$, and making use of Equation 11 yields

$$
\lambda_{k}=-\frac{\gamma_{k}{ }^{r_{k}}}{\sum_{i=1}^{S} \gamma_{i}{ }^{r}{ }_{i}}, k=2,3, \ldots, S
$$

Finally, setting $\partial \mathrm{F} / \partial \mathrm{P}_{\text {in }}=0$ and substituting Equations 11 and 14 results in

$$
\frac{\gamma_{1}}{x_{1}^{2}} \frac{d X_{1}}{d P_{i n}}+\sum_{k=2}^{S} \frac{\gamma_{k}}{\beta_{k} x_{k}^{2}} \frac{d x_{k}}{d P_{i n}}=0
$$

which can be written as

$$
\frac{d}{d P_{i n}}\left[\sum_{k=1}^{S} \frac{\gamma_{k}}{\beta_{k} x_{k}}\right]=0
$$


Substitution of Equation 8 into Equation 16 leads to the condition

$$
\frac{\mathrm{d}}{\mathrm{dP}}\left[\frac{\mathrm{kB}\left(\mathrm{T}_{\mathrm{u}} \mathrm{G}+\mathrm{T}_{\mathrm{d}}^{\mathrm{eq}}\right)+\mathrm{IM}_{\mathrm{c}}}{\mathrm{C}_{\mathrm{a}}}\right]=0
$$

where

$$
\mathrm{T}_{\mathrm{d}}^{\mathrm{eq}}=\frac{\sum_{\mathrm{k}=1}^{\mathrm{S}} \gamma_{\mathrm{k}} \mathrm{T}_{\mathrm{dk}} / \beta_{\mathrm{k}}}{\sum_{\mathrm{k}=1}^{S} \gamma_{\mathrm{k}} / \beta_{\mathrm{k}}}
$$

Equation 17 may be recognized as the CNR maximizing condition for a system of equal amplitude carriers in which the common carrier power is $\mathrm{C}_{\mathrm{a}}$, the (common) earth station $\mathrm{G} / \mathrm{T}$ is represented by $\mathrm{T}_{\mathrm{d}}^{\mathrm{eq}}$ and the same CNR is required at all stations. The optimum value of $P_{\text {in }}$ can therefore be found by the usual technique of searching for the minimum value of the quantity in brackets through a tradeoff of thermal vs. IM noise. Once $\mathrm{P}_{\text {in }}$ has been determined, the optimum value of $r_{k}, k=2,3, \quad, S$, can be found from Equations 8 and 11 .

Note that $\mathrm{T}_{\mathrm{d}}^{\mathrm{eq}}$ is a weighted average of the downlink noise temperatures for the various station types. In particular, when $\beta_{\mathrm{k}}=1, \mathrm{k}=1, \ldots, \mathrm{S}$, the weights are in direct proportion to the number of carriers received at each station type.

\section{TWO-LEVEL SYSTEM}

For the two-level system, notation will be introduced which is suggestive of the relative carrier sizes. The subscript $\mathrm{L}$ will be used to denote a quantity relating to the large carriers (i.e., those received at the small $\mathrm{G} / \mathrm{T}$ stations), and the subscript $\mathrm{S}$ will be used to denote a quantity relating to the small carriers." If the small $\mathrm{G} / \mathrm{T}$ stations are designated as type 1 stations and the large G/T stations as type 2 stations, the fractional number of large and small carriers is given by

$$
\begin{aligned}
& \gamma_{L}=\gamma_{11}+\frac{1}{2} \gamma_{12} \\
& \gamma_{S}=\gamma_{22}+\frac{1}{2} \gamma_{12}
\end{aligned}
$$

\footnotetext{
* It is conceivable, if the CNR requirement at the large stations is sufficiently greater than that at the small stations, for the large carriers in an optimally designed system to be received at the large stations. However, this possibility is not likely to occur in practice and can safely be ignored.
} 
The condition defining the optimum transponder backoff is again provided by Equation 17, where $T_{d}^{\text {eq }}$ is now given by

$$
T_{d}^{e q}=\frac{\gamma_{L} T_{d l}+\left(\gamma_{S} / \beta\right) T_{d 2}}{\gamma_{L}+\gamma_{S} / \beta}
$$

and $\beta$ is defined by

$$
\left(\frac{\mathrm{C}}{\mathrm{N}}\right)_{\mathrm{S}}=\frac{1}{\beta}\left(\frac{\mathrm{C}}{\mathrm{N}}\right)_{\mathrm{L}}
$$

With the optimum backoff determined, the ratio of the power in a single small carrier to that in a single large carrier, $r$, can be found from the set of relations

$$
\begin{gathered}
X_{L}=\frac{C_{a}}{k B\left(T_{u} G+T_{d l}\right)+I M_{C}} \\
X_{S}=\frac{C_{a}}{k B\left(T_{u} G+T_{d 2}\right)+I M_{C}} \\
r=\frac{X_{L}}{\beta X_{S}}
\end{gathered}
$$

With the transponder backoff and the small-to-large carrier power ratio chosen in accordance with Equation 17 and Equations 23 through 25, the CNR for the two carrier types is given by

$$
\begin{aligned}
& \left(\frac{C}{N}\right)_{L}=\frac{X_{L}}{Y_{L}+\gamma_{S}^{r}} \\
& \left(\frac{C}{N}\right)_{S}=\frac{r X_{S}}{\gamma_{L}+\gamma_{S}^{r}}
\end{aligned}
$$

Performance of an optimally designed two-level system will be illustrated for a typical set of C-band parameters. The large station $\mathrm{G} / \mathrm{T}$ is fixed at $30 \mathrm{~dB} /{ }^{\circ} \mathrm{K}$, while the small station $\mathrm{G} / \mathrm{T}$ is regarded as a parameter. The intermodulation characteristics used in the optimization are those of the Hughes $275 \mathrm{H}$ TWT. Curves of carrier-to-IM (C/IM) ratio vs. backoff for this TWT, analytically derived from the single-carrier transfer characteristic, can be found in Reference 1. A useful transponder bandwidth of $36 \mathrm{MHz}$ and a 10 percent 
guard band between carriers have been assumed. The transponder saturation EIRP has been taken as $33 \mathrm{dBW}$, the corresponding input saturation power as $-102 \mathrm{dBW}$, and the satellite receive system noise temperature as $30 \mathrm{~dB}-{ }^{\circ} \mathrm{K}$.

The maximum attainable CNR (at the center carrier position) for a fully loaded transponder with continuously transmitted carriers is shown in Figure 1 as a function of the small station $\mathrm{G} / \mathrm{T}$, for several values of $\gamma_{\mathrm{L}}$. Equal CNR requirements have been assumed for the large and small carriers. The transponder backoff and the large-to-small-carrier power ratio required to achieve the performance shown in Figure 1 are given in Figures 2 and 3.

The impro vement in system performance afforded by a two-level system, over that for a uniform carrier system, can be found by comparing the curve for the appropriate value of $\gamma_{L}$ in Figure 1 with that for $\gamma_{L}=1.0$. This can be seen by observing that, for $\gamma=1.0$, all traffic is conducted between pairs of small stations. The carriers are therefore all the same size and the achievable CNR is a function only of the small station G/T. The latter pair of conditions also characterizes a system in which the carriers are constrained to have the same power, regardless of the network traffic pattern. Thus, the $\gamma=1.0$ curve specifies the attainable performance of a uniform carrier system under any set of traffic conditions.

Consider a system of voice-activated carriers with a CNR requirement of $12 \mathrm{~dB}$. With a voice activity factor of 0.4 , the transponder capacity is the same as that for a system of uninterrupted carriers with a $4 \mathrm{~dB}$ lower CNR requirement. (This follows from the fact that, with the TWT backoff and the value of $r$ unchanged, the power in each carrier of the activated system can be made $4 \mathrm{~dB}$ larger than the power in the corresponding carrier type of the nonactivated system, without affecting the average IM power falling in a given carrier slot.) Accordingly, an $8 \mathrm{~dB}$ CNR requirement will be imposed on the curves in Figure 1. If $\gamma_{\mathrm{L}}=0.2$, for example, full transponder loading can be achieved with a small station $\mathrm{G} / \mathrm{T}$ of $17.7 \mathrm{~dB} /{ }^{\circ} \mathrm{K}$. By contrast, the $\mathrm{G} / \mathrm{T}$ requirement in a system of uniform carriers is $23.9 \mathrm{~dB} /{ }^{\circ} \mathrm{K}$, a difference of $6.2 \mathrm{~dB}$. If $\gamma_{\mathrm{L}}=0.5$, a two-level system provides an advantage of $2.6 \mathrm{~dB}$ in terms of the required $\mathrm{G} / \mathrm{T}$. This advantage increases to a maximum of $3 \mathrm{~dB}$ as the CNR requirement is reduced or the transponder bandwidth occupancy is decreased from $36 \mathrm{MHz}$.

\section{CONCLUSIONS}

A method of assigning carrier power levels in an SCPC system employing a mixture of earth station sizes has been developed which maximizes the satellite channel capacity or, if the system is bandwidth limited, maximizes the system power margin. The technique is based on a random assignment of carrier frequencies and is therefore ideally suited for use in a demand assignment system. To implement such a system, the channel units must be 
provided with a commendable gain adjustment, and the system software must be designed to exercise gain control in conjunction with carrier frequency assignment.

\section{REFERENCE}

1. Horstein, M. and LaFlame, D. T. , "Intermodulation Spectra For Two SCPC Systems," IEEE Transactions on Communications, Volume COM-25, No. 9, September 1977, pp. 990-994.

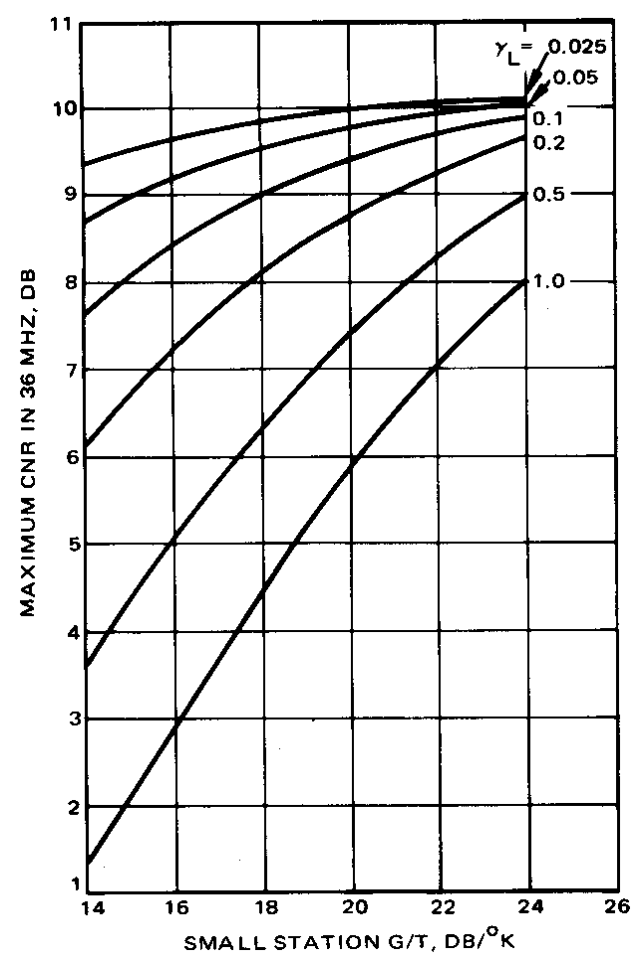

FIGURE 1. OPTIMUM SYSTEM PERFORMANCE

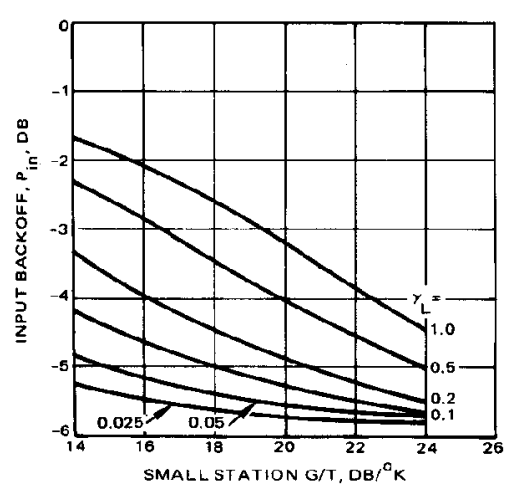

FIGURE 3. REQUIRED CARRIER POWER RATIO

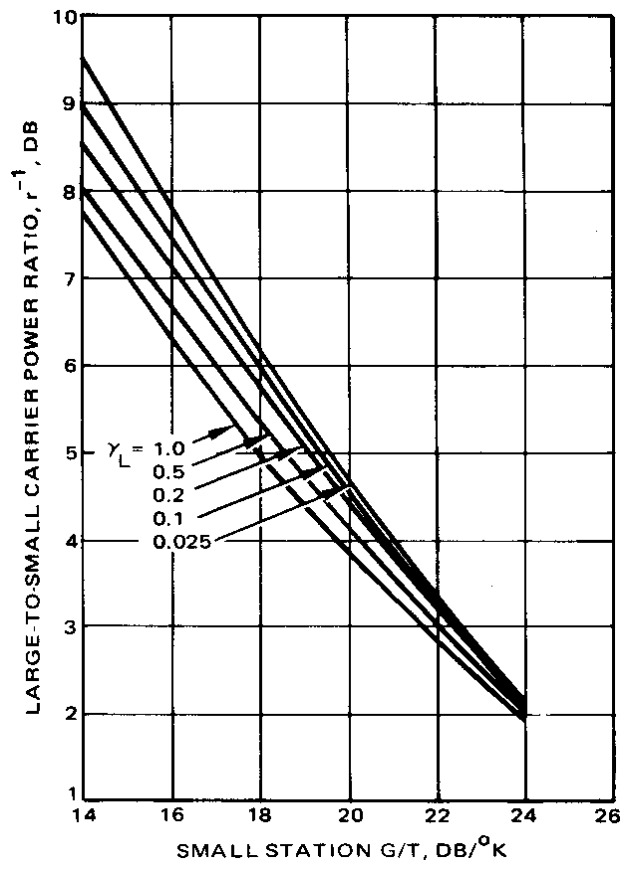

FIGURE 2. REQUIRED TRANSPONDER BACKOFF 